\title{
Safety and Risk of Medication Overuse Headache in Lasmiditan and Second-Generation Gepants: A Rapid Review
}

\author{
Flavia Lo Castro (D) \\ Simona Guerzoni \\ Lanfranco Pellesi (iD) ${ }^{2}$ \\ 'Medical Toxicology, Headache and Drug \\ Abuse Research Center, Department of \\ Specialized Medicine, AOU Policlinico di \\ Modena, Modena, Italy; ${ }^{2}$ Danish \\ Headache Center, Department of \\ Neurology, Rigshospitalet Glostrup, \\ Faculty of Health and Medical Sciences, \\ University of Copenhagen, Copenhagen, \\ Denmark
}

\begin{abstract}
The treatment of migraine is often complicated by insufficient headache relief, a miscellany of side effects and the risk of developing Medication Overuse Headache $(\mathrm{MOH})$. Novel acute therapies have been recently developed and are now in the early postmarketing phase. Lasmiditan is a highly selective serotonin receptor agonist that binds to the $5-\mathrm{HT}_{1 \mathrm{~F}}$ receptor, while ubrogepant and rimegepant antagonize the calcitonin gene-related peptide receptor. All three medications are now prescribed in a real-world setting, and an adequate level of knowledge is the starting point for rational use. In this rapid systematic review, we have established what is known about lasmiditan, ubrogepant and rimegepant, highlighting the most relevant safety aspects available from published studies and speculating about their risk of $\mathrm{MOH}$.
\end{abstract}

Keywords: CGRP, headache, migraine, $\mathrm{MOH}$, triptans

\section{Introduction}

Migraine is a common pain condition and the second most disabling disorder worldwide. ${ }^{1}$ The acute treatment ranges from the use of simple analgesics, such as paracetamol or nonsteroidal anti-inflammatory drugs (NSAIDs), to migrainespecific therapies, including ergotamine and its derivatives or triptans. ${ }^{2}$ Not all patients respond to treatments, experiencing side effects or an insufficient relief from their attacks. ${ }^{3}$ Triptans are contraindicated in patients with cardiovascular diseases, hypertension, or during pregnancy, and their use is mostly off-label in pediatric patients. ${ }^{4}$ NSAIDs are not indicated in patients with peptic ulcer disease, inflammatory bowel diseases, renal dysfunction, and those on concomitant anticoagulants. ${ }^{5}$ In addition, the frequent use of any acute treatment for migraine can paradoxically worsen the headache itself, leading to a secondary pain condition called medication overuse headache $(\mathrm{MOH})$. In the International Classification, patients with $\mathrm{MOH}$ are defined with a pre-existing headache, who develop a significant worsening in association with the use of acutely acting medications at least 15 days per month (simple analgesics) or 10 days per month (triptans, ergot derivatives, opioids or combination analgesics). ${ }^{6} \mathrm{MOH}$ is a common cause of chronic daily headache and affects $1-2 \%$ of the population worldwide. $^{7}$

Based on the premise that the calcitonin gene-related peptide (CGRP) plays a key role in the pathophysiology of migraine, a new generation of selective treatments has
Correspondence: Lanfranco Pellesi Danish Headache Center, Department of Neurology, Rigshospitalet Glostrup Faculty of Health and Medical Sciences, University of Copenhagen, Copenhagen, Denmark

Tel +45 53803056

Email lanfranco.pellesi@gmail.com 
Table I Safety Profile of Lasmiditan, Ubrogepant and Rimegepant Available from Published Trials

\begin{tabular}{|c|c|c|c|}
\hline & Lasmiditan & Ubrogepant & Rimegepant \\
\hline Target & $5-\mathrm{H} \mathrm{T}_{\mathrm{IF}}$ receptors & CGRP receptor & CGRP receptor \\
\hline Formulation & Oral tablet & Oral tablet & Oral tablet \\
\hline Dosage & 50,100 and $200 \mathrm{mg}$ & 50 and $100 \mathrm{mg}$ & $75 \mathrm{mg}$ \\
\hline $\begin{array}{l}\text { Most common treatment-emergent adverse } \\
\text { events }\end{array}$ & $\begin{array}{l}\text { Dizziness } \\
\text { Fatigue } \\
\text { Nausea } \\
\text { Paraesthesia } \\
\text { Somnolence }\end{array}$ & $\begin{array}{l}\text { Dizziness } \\
\text { Nausea } \\
\text { Somnolence }\end{array}$ & $\begin{array}{c}\text { Dizziness } \\
\text { Nausea } \\
\text { Urinary tract infection }\end{array}$ \\
\hline Treatment-related serious adverse events & $\begin{array}{l}\text { Serotonin syndrome }(\mathrm{n}=\mathrm{I})^{23} \\
\text { Driving impairment up to } 8 \text { hours after } \\
\text { dosing }^{28}\end{array}$ & $\begin{array}{l}\text { Seizure }(n=1)^{34} \\
\text { Sinus tachycardia } \\
\qquad(\mathrm{n}=1)^{36}\end{array}$ & None \\
\hline
\end{tabular}

emerged. ${ }^{8}$ These therapies include: (i) lasmiditan; (ii) second-generation gepants, or small-molecule antagonists that antagonize the CGRP receptor (Table 1). Lasmiditan belongs to the serotonin $(5-\mathrm{HT}) 5-\mathrm{HT}_{1 \mathrm{~F}}$ receptor agonists, which lead to the inhibition of the CGRP release in the trigeminovascular signaling pathways. ${ }^{9}$ Ubrogepant and rimegepant are second-generation gepants that have overcome the liver toxicity associated with the first generation. Lasmiditan, ubrogepant and rimegepant have been recently approved by the Food and Drug Administration (FDA) for adult patients with migraine and are now in the early post-marketing phase. An enhanced focus on safety improves the appropriateness of the initial prescribing and provides an opportunity to minimize potential adverse drug events.

In this rapid systematic review, we aimed to summarize the clinical safety of lasmiditan, ubrogepant and rimegepant from published studies, with a section discussing how these drugs are expected to relate to $\mathrm{MOH}$.

\section{Materials and Methods}

Rapid reviews are an emerging type of knowledge synthesis used to inform health-related discussions, especially when information needs are immediate. ${ }^{10-12}$ The rapid review method used is similar to previously published reviews. ${ }^{12,13}$ Briefly, we focused on English, peer-reviewed full abstracts in PubMed published before 31 June 2021 and used the following non-MESH keywords: lasmiditan, LY573144, ubrogepant, MK-1602, rimegepant and BHV3000. The search was run in
PubMed because of time constraints and because it is the most widely searched database for health-related topics. The initial search (July 2021) yielded 290 titles or abstracts. Similar keywords were also used in a brief online grey literature search, which retrieved an additional list of in-progress trials. All findings were screened for duplicates and relevancy concerning the clinical effects of lasmiditan, ubrogepant or rimegepant in patients with migraine and/or their potential to induce $\mathrm{MOH}$. Review articles were further examined to find any primary sources that may have been missed in the searches.

\section{Lasmiditan}

Currently, triptans are considered first-line treatments for moderate-to-severe migraine. However, a lack of response is visible in $25-30 \%$ of patients, and triptans are contraindicated in patients with cardiovascular diseases, as they have potential vasoconstrictive effects on cerebral and coronary arteries. ${ }^{14,15}$ This fostered the search for more specific therapies, leading to the development of selective $5-\mathrm{HT}_{1 \mathrm{~F}}$ receptor agonists, also called ditans. Lasmiditan is a lipophilic ditan that acts on the trigeminal system without causing vasoconstriction, ${ }^{16}$ approved by the FDA in October 2019. The recommended dosage is $50 \mathrm{mg}$, $100 \mathrm{mg}$ or $200 \mathrm{mg}$ taken orally, as needed. Upon oral administration, lasmiditan is rapidly absorbed, reaching a peak plasma concentration of approximately 1.8 hours. Taking lasmiditan with a high-fat meal may prolong the time to reach the maximum plasma 
concentration, but it is not expected to be clinically significant. ${ }^{17}$ The biological half-life of lasmiditan is $5.7 \mathrm{~h}$, the binding to blood proteins is around $60 \%$. Lasmiditan is primarily eliminated via metabolism, whereas renal excretion plays only a minor role in drug clearance. ${ }^{17}$ When administered orally, lasmiditan reduced migraine pain and the most bothersome symptoms within two hours. ${ }^{18-20}$ Compared to placebo, treatment-emergent related adverse events (TEAEs) were reported more frequently within 48 hours in patients assigned to lasmiditan. ${ }^{21}$ TEAEs were dosedependent, usually mild to moderate in intensity, and self-limiting. ${ }^{22}$ The onset occurred approximately 30 50 minutes after administration, with the frequency decreasing with subsequent attacks. ${ }^{22,23}$ The most common adverse events (AEs) were neurological events, including dizziness (14.7\%), and paraesthesia $(5.7 \%){ }^{21}$ Other common events were somnolence $(5.5 \%)$, fatigue $(3.8 \%)$, and nausea $(3.4 \%) .^{21}$ They were similar between patients using and not using preventive medications and appeared independent of comorbid conditions. ${ }^{24,25}$ Among serious TEAEs, only a single case of serotonin syndrome was considered related to lasmiditan. ${ }^{23}$ In vitro, lasmiditan exhibited inhibition of intestinal P-glycoprotein, indicating a potential interaction in vivo. ${ }^{26}$ In a Phase I study conducted in 44 healthy subjects, coadministration of lasmiditan in the presence of propranolol decreased heart rate shortly after dosing while increasing blood pressure relative to propranolol alone. ${ }^{27}$ The cardiovascular parameters returned to baseline levels within 3 hours, whereas the heart rate remained significantly lower over the entire 12-hour post dose. In two randomized, placebo-controlled studies, lasmiditan showed impaired simulated driving performance at 1.5 -hours post-dose, suggesting that individuals taking lasmiditan should not engage in potentially hazardous activities requiring complete mental alertness, such as driving a motor vehicle or operating machinery, for at least 8 hr after administration. ${ }^{28}$ Lasmiditan is safe and well tolerated in patients with migraine aged 6 to 17 years, ${ }^{29}$ and clinical trials investigating the efficacy of such a population are currently underway (NCT04396236 and NCT04396574).

\section{Ubrogepant}

Ubrogepant is the first oral CGRP receptor antagonist approved for the acute treatment of migraine. ${ }^{30}$ The recommended dosage is $50 \mathrm{mg}$ or $100 \mathrm{mg}$, up to two doses per day with at least 2 hours apart. Ubrogepant is rapidly absorbed and the maximum concentration of plasma is achieved after 1.5 hours. ${ }^{31}$ Consuming ubrogepant with fatty foods may extend the absorption process, but no substantial clinical effects are expected. The half-life is around 6 hours, the apparent central volume of distribution is high $(350 \mathrm{~L})$, the binding to blood proteins is $87 \% .{ }^{31}$ Ubrogepant is metabolized primarily by the cytochrome P450 3A4 (CYP3A4), and it is mainly excreted in the feces. The efficacy and safety of ubrogepant was demonstrated in three randomized, double-blind, placebo-controlled trials. ${ }^{32-34}$ TEAEs were mainly mild or moderate in intensity, with no significant difference between ubrogepant and placebo within 48 hours post-dose and for 30 days followup. ${ }^{35}$ The long-term safety and tolerability of ubrogepant given as 1 or 2 doses per attack was further investigated in an open-label, 52-week trial. ${ }^{36}$ Ubrogepant-related AEs occurred approximately in $10 \%$ of participants, with the most common being nausea $(1.5 \%$ and $1.7 \%$ with ubrogepant 50 and $100 \mathrm{mg}$, respectively), dizziness (0.5\% and $1.5 \%)$, and somnolence $(1.5 \%$ and $1.2 \%){ }^{36}$ No increase in the incidence of TEAEs was observed with an increased number of attacks treated per month. Discontinuation due to AEs was reported in $2-3 \%$ of ubrogepant-treated participants. ${ }^{36}$ A single serious adverse event (sinus tachycardia) was considered related to ubrogepant, this event occurred in a participant with a history of supraventricular tachycardia with ablation. ${ }^{36}$ The participant continued taking ubrogepant without further complications. No safety concerns were identified based on laboratory or vital sign findings, or by the presence of major cardiovascular risk factors. ${ }^{37}$ Concomitant use of ubrogepant with strong CYP3A4 inducers (eg, rifampin, phenytoin, barbiturates) should be avoided due to a possible decreased efficacy. Ubrogepant should not be administered with potent CYP3A4 inhibitors (eg, clarithromycin, ketoconazole, itraconazole) as these drugs may cause a significant increase in ubrogepant plasma concentration. The manufacturer recommends an initial ubrogepant dose of $100 \mathrm{mg}$ when coadministered with moderate or weak CYP3A4 inducers, or a starting dose of $50 \mathrm{mg}$ when ubrogepant is used concomitantly with moderate or weak inhibitors of CYP3A4. Ubrogepant showed no clinically relevant signal of hepatotoxicity following intermittent, high- 
frequency dosing, or clinically significant drug-drug interactions with erenumab, galcanezumab, paracetamol, naproxen, sumatriptan and components of an oral contraceptive. $^{38-42}$

\section{Rimegepant}

Rimegepant is the first and only CGRP receptor antagonist available in the form of orally disintegrating tablets. $^{43}$ The recommended dose is $75 \mathrm{mg}$ as needed, no more than one tablet per day. After administration, the maximum plasma concentration is achieved after 1.5 hours. ${ }^{44}$ When administered with a high-fat meal, the absorption is delayed, the maximum plasma concentration is reduced by $42-53 \%$. The half-life is around 11 hours, the volume of distribution and the plasma protein binding are high $\left(120 \%\right.$ and $96 \%$, respectively). ${ }^{44}$ Metabolism of rimegepant is mainly mediated by the CYP3A4 isoenzyme and to a lesser extent by the cytochrome P450 2C9 (CYP2C9). About 77\% of rimegepant is excreted, primarily unchanged in the urine. Rimegepant demonstrated efficacy and safety in three randomized, double-blind, placebo-controlled trials conducted in patients with migraine. ${ }^{45-47}$ Most TEAEs in rimegepant-treated individuals were mild to moderate in intensity, with the most common being nausea (1.6\%), followed by urinary tract infection $(1.5 \%)$ and dizziness $(0.8 \%) .{ }^{48}$ No serious AEs related to rimegepant, or deaths were reported. Although previous studies with first-generation CGRP receptor antagonists, such as olcegepant, found that the most relevant $\mathrm{AE}$ was hepatotoxicity, ${ }^{49}$ a recent meta-analysis reported that rimegepant is not associated with an increased liver damage. ${ }^{48}$ Taken every other day, rimegepant was also safe and effective as a preventive treatment for migraine, with no safety issues reported. ${ }^{50}$ No treatment-related serious AEs were reported in the rimegepant group, while $2 \%$ of participants who received rimegepant discontinued due to an adverse event. ${ }^{50}$ The FDA accepted the supplemental New Drug Application for the preventive treatment of migraine in October 2020 and rimegepant has been recently approved for dual therapy for acute and preventive treatment of migraine in adults. The concomitant administration of rimegepant with strong or moderate inhibitors and inducers of CYP3A4 should be avoided, whereas the induction or inhibition of CYP2C9 is not expected to have a significant effect on rimegepant exposure. $^{51}$ No dosage adjustment is required for patients with mild, moderate, or severe renal impairment, or patients with mild or moderate hepatic injury. However, its use should be avoided in patients with severe hepatic impairment. ${ }^{51}$ Rimegepant is well tolerated when used concomitantly with sumatriptan, erenumab, fremanezumab and galcanezumab. ${ }^{52,53}$ Two multicenter studies are currently ongoing to evaluate the tolerability and efficacy of rimegepant in children and adolescents for the acute treatment of migraine (NCT04649242 and NCT04743141).

\section{Is There a Risk for MOH?}

The relationship between the new therapies for migraine and $\mathrm{MOH}$ is being discussed. ${ }^{54}$ The novelty of lasmiditan, ubrogepant and rimegepant makes it impossible to draw conclusions based upon available clinical trials, often of a relatively short duration and not assessing their risk for $\mathrm{MOH}$. Usually, the safety and tolerability of a medication overuse use is not assessed before the drug is prescribed. To speculate about the matter, the findings in animal studies might be worth considering. Animal models of $\mathrm{MOH}$ have been developed with the aim to comprehend the neural adaptations due to the repeated overuse of analgesic medications. ${ }^{55}$ These models exhibit phenotypes that relate to $\mathrm{MOH}$, such as mechanical allodynia, hyperalgesia and nociceptive behaviours. An increased level of CGRP was, in some studies, associated with preclinical MOH and blocking the CGRP pathway with an antibody prevented cutaneous allodynia in rodents sensitized with sumatriptan and morphine. ${ }^{55-59}$ At the same time, the administration of monoclonal antibodies antagonising the CGRP pathway was effective and reduced headache in patients with $\mathrm{MOH}$ in clinical trials and in the real-world setting. ${ }^{60-65}$ Targeting the CGRP signaling for the acute treatment of migraine may well be a promising approach to maintain a low risk for $\mathrm{MOH}$ development. When administered on a regular basis, ubrogepant and olcegepant did not induce cutaneous allodynia or neuroplastic changes in trigeminal sensory afferents in rats, ${ }^{66,67}$ whereas a persistent exposure to lasmiditan induced cutaneous allodynia and neuroplastic changes in mice, including an increased expression of CGRP in trigeminal sensory afferents. ${ }^{66,68}$ Although triptans and lasmiditan act on different receptor subtypes, they inhibit postsynaptic adenosine 3',5'-cyclic monophosphate (cAMP) signaling cascades and may have a different risk of inducing 


\section{Treatment-emergent AEs (\%)}

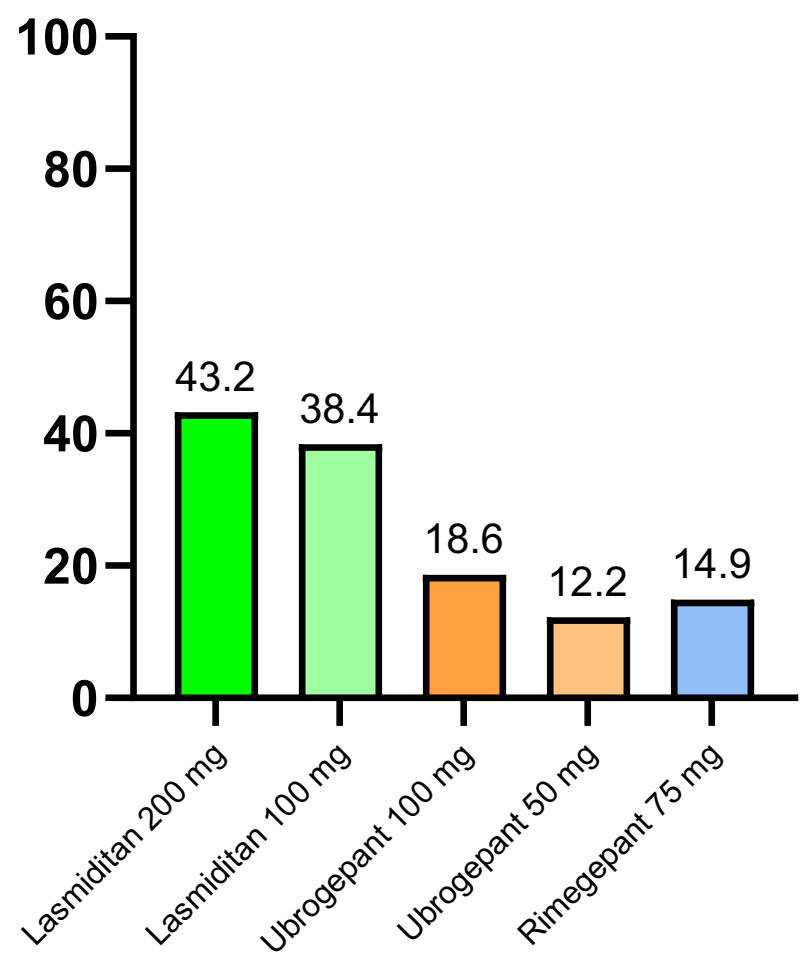

Figure I Mean percentage of treatment-emergent adverse events (AEs) of lasmiditan, ${ }^{18-20}$ ubrogepant ${ }^{32-34}$ and rimegepant ${ }^{45-47}$ from available clinical studies.

$\mathrm{MOH}$ compared to second generation gepants. However, our speculations require caution. Rather than measuring an increased frequency of headache, preclinical models only focus on the effects induced by $\mathrm{MOH}-i n d u c i n g$ drugs. Patients with migraine may have a specific susceptibility to $\mathrm{MOH}$, that is not addressed in animals. Moreover, other studies questioned the role of CGRP in the pathophysiology of MOH. ${ }^{69,70}$ Currently, there are no data indicating that the chronic administration of lasmiditan induces $\mathrm{MOH}$. If established clinically, gepants might be of great use in patients who have a history of $\mathrm{MOH}$ or are at risk of developing $\mathrm{MOH}$, including those with frequent migraine attacks.

\section{Conclusions}

Clinical trials with lasmiditan, ubrogepant and rimegepant have shown promising results in aborting migraine attacks. Their safety profile appears favourable across all the clinical studies conducted. Lasmiditan is associated with a higher rate of TEAEs, while preclinical studies suggest it may be associated with a certain risk for $\mathrm{MOH}$

\section{Treatment-related AEs (\%)}

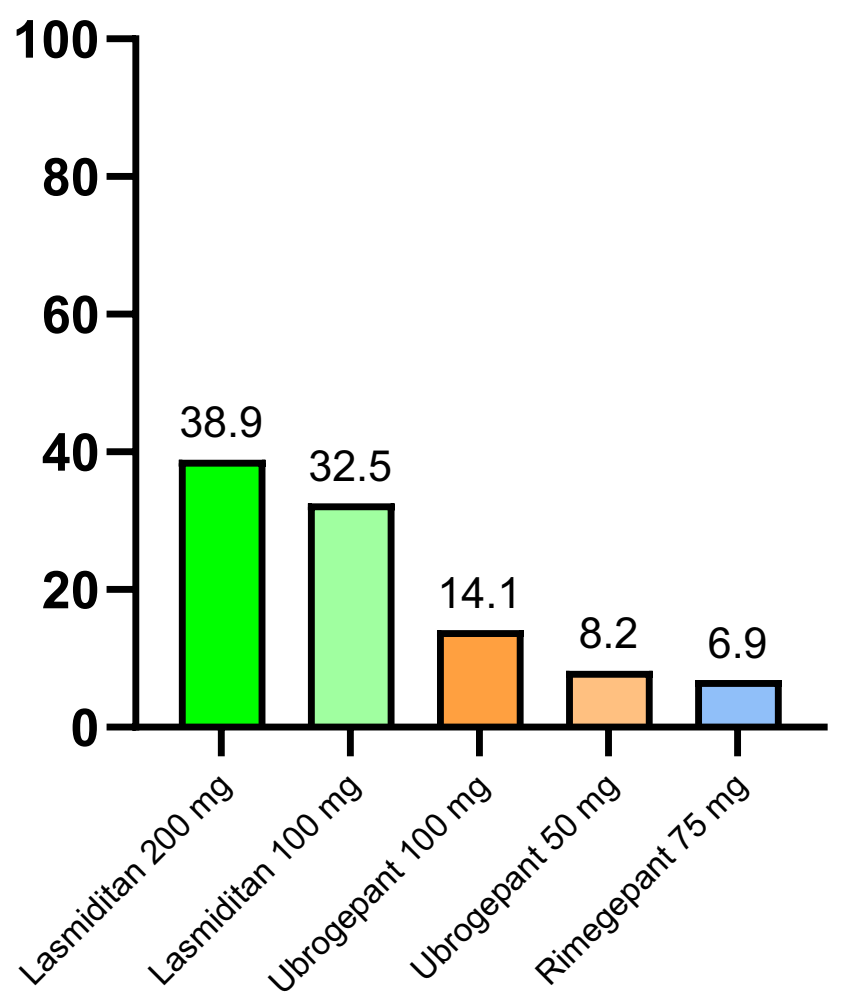

Figure 2 Mean percentage of treatment-related adverse events (AEs) of lasmiditan, ${ }^{18-20}$ ubrogepant $^{32-34}$ and rimegepant ${ }^{45-47}$ from available clinical studies.

(Figures 1 and 2). However, the relationship of lasmiditan, ubrogepant and rimegepant with $\mathrm{MOH}$ requires more research. The real-world population may be particularly susceptible to side effects, especially when the intake is frequent and prolonged over time. Future evaluations will better determine the chronic effects of $5-\mathrm{HT}_{1 \mathrm{~F}}$ receptor agonists and CGRP receptor antagonists.

\section{Abbreviations}

5-HT, serotonin; AE, adverse event; cAMP, adenosine 3',5'-cyclic monophosphate; CGRP, calcitonin generelated peptide; CYP2C9, cytochrome P450 2C9; CYP3A4, cytochrome P450 3A4; FDA, Food and Drug Administration; $\mathrm{MOH}$, medication overuse headache; NSAID, nonsteroidal anti-inflammatory drug; TEAE, treatment-emergent related adverse event.

\section{Disclosure}

R Simona Guerzoni reports personal fees from TEVA, personal fees from ALLERGAN, personal fees from NOVARTIS, personal fees from LILLY, during the 
conduct of the study. The authors report no other conflicts of interest in this work.

\section{References}

1. Burch RC, Buse DC, Lipton RB. Migraine: epidemiology, burden, and comorbidity. Neurol Clin. 2019;37(4):631-649. doi:10.1016/j. ncl.2019.06.001

2. Gilmore B, Michael M. Treatment of acute migraine headache. Am Fam Physician. 2011;83(3):271-280.

3. González-Hernández A, Marichal-Cancino BA, MaassenVanDenBrink A, Villalón CM. Side effects associated with current and prospective antimigraine pharmacotherapies. Expert Opin Drug Metab Toxicol. 2018;14 (1):25-41. doi:10.1080/17425255.2018.1416097

4. Rapoport AM, Tepper SJ, Bigal ME, Sheftell FD. The triptan formulations: how to match patients and products. CNS Drugs. 2003;17 (6):431-447. doi:10.2165/00023210-200317060-00005

5. Ong JJY, De Felice M. Migraine treatment: current acute medications and their potential mechanisms of action. Neurotherapeutics. 2018;15 (2):274-290. doi:10.1007/s13311-017-0592-1

6. Headache classification committee of the International Headache Society (IHS) the international classification of headache disorders, 3rd edition. Cephalalgia. 2018;38(1):1-211. doi:10.1177/033310 2417738202

7. Diener HC, Holle D, Solbach K, Gaul C. Medication-overuse headache: risk factors, pathophysiology and management. Nat Rev Neurol. 2016;12(10):575-583. doi:10.1038/nrneurol.2016.124

8. Ashina M, Ropper AH. Migraine. $N$ Engl J Med. 2020;383 (19):1866-1876. doi:10.1056/NEJMra1915327

9. Labastida-Ramírez A, Rubio-Beltrán E, Haanes KA, et al. Lasmiditan inhibits calcitonin gene-related peptide release in the rodent trigeminovascular system. Pain. 2020;161(5):1092-1099. doi:10.1097/j. pain.0000000000001801

10. Watt A, Cameron A, Sturm L, et al. Rapid reviews versus full systematic reviews: an inventory of current methods and practice in health technology assessment. Int J Technol Assess Health Care. 2008;24(2):133-139. doi:10.1017/S0266462308080185

11. Ganann R, Ciliska D, Thomas H. Expediting systematic reviews: methods and implications of rapid reviews. Implement Sci. 2010;5 (1):56. doi:10.1186/1748-5908-5-56

12. Khangura S, Konnyu K, Cushman R, et al. Evidence summaries: the evolution of a rapid review approach. Syst Rev. 2012;1(1):10. doi:10.1186/2046-4053-1-10

13. Lal S, Adair CE. E-mental health: a rapid review of the literature. Psychiatr Serv. 2014;65(1):24-32. doi:10.1176/appi. ps.201300009

14. MaassenVanDenBrink A, van den Broek RW, de Vries R, Bogers AJ, Avezaat CJ, Saxena PR. Craniovascular selectivity of eletriptan and sumatriptan in human isolated blood vessels. Neurology. 2000;55 (10):1524-1530. doi:10.1212/WNL.55.10.1524

15. MacIntyre PD, Bhargava B, Hogg KJ, Gemmill JD, Hillis WS. Effect of subcutaneous sumatriptan, a selective 5HT1 agonist, on the systemic, pulmonary, and coronary circulation. Circulation. 1993;87 (2):401-405. doi:10.1161/01.CIR.87.2.401

16. Lucaites VL, Krushinski JH, Schaus JM, Audia JE, Nelson DL. [3H] LY334370, a novel radioligand for the 5-HT1F receptor. II. Autoradiographic localization in rat, Guinea pig, monkey and human brain. Naunyn Schmiedebergs Arch Pharmacol. 2005;371 (3):178-184. doi:10.1007/s00210-005-1036-8

17. Ferrari A, Rustichelli C. Rational use of lasmiditan for acute migraine treatment in adults: a narrative review. Clin Ther. 2021;43 (4):654-670. doi:10.1016/j.clinthera.2021.01.020
18. Färkkilä M, Diener HC, Gèraud G, et al. Efficacy and tolerability of lasmiditan, an oral $5-\mathrm{HT}_{1 \mathrm{~F}}$ receptor agonist, for the acute treatment of migraine: a Phase 2 randomised, placebo-controlled, parallel-group, dose-ranging study. Lancet Neurol. 2012;11(5):405-413. doi:10.10 16/S1474-4422(12)70047-9

19. Kuca B, Silberstein SD, Wietecha L, et al. Lasmiditan is an effective acute treatment for migraine: a Phase 3 randomized study. Neurology. 2018;91(24):e2222-e2232. doi:10.1212/WNL.0000000000006641

20. Goadsby PJ, Wietecha LA, Dennehy EB, et al. Phase 3 randomized, placebo-controlled, double-blind study of lasmiditan for acute treatment of migraine. Brain. 2019;142(7):1894-1904. doi:10.1093/brain/ awz134

21. Krege JH, Rizzoli PB, Liffick E, et al. Safety findings from Phase 3 lasmiditan studies for acute treatment of migraine: results from SAMURAI and SPARTAN. Cephalalgia. 2019;39(8):957-966. doi:10.1177/0333102419855080

22. Brandes JL, Klise S, Krege JH, et al. Interim results of a prospective, randomized, open-label, phase 3 study of the long-term safety and efficacy of lasmiditan for acute treatment of migraine (the GLADIATOR study). Cephalalgia. 2019;39(11):1343-1357. doi:10. 1177/0333102419864132

23. Ashina M, Reuter U, Smith T, et al. Randomized, controlled trial of lasmiditan over four migraine attacks: findings from the CENTURION study. Cephalalgia. 2021;41(3):294-304. doi:10.11 $77 / 0333102421989232$

24. Loo SL, Ailani J, Schim J, et al. Efficacy and safety of lasmiditan in patients using concomitant migraine preventive medications: findings from SAMURAI and SPARTAN, two randomized phase 3 trials. J Headache Pain. 2019;20(1):84. doi:10.1186/s10194-019-1032-x

25. Clemow DB, Baygani SK, Hauck PM, Hultman CB. Lasmiditan in patients with common migraine comorbidities: a post hoc efficacy and safety analysis of two phase 3 randomized clinical trials. Curr Med Res Opin. 2020;36(11):1791-1806. doi:10.1080/03007995.2020.1808780

26. Szkutnik-Fiedler D. Pharmacokinetics, pharmacodynamics and drug-drug interactions of new anti-migraine drugs-lasmiditan, gepants, and Calcitonin-Gene-Related Peptide (CGRP) receptor monoclonal antibodies. Pharmaceutics. 2020;12(12):1180. doi:10. 3390/pharmaceutics 12121180

27. Tsai M, Case M, Ardayfio P, Hochstetler H, Wilbraham D. Effects of lasmiditan on cardiovascular parameters and pharmacokinetics in healthy subjects receiving oral doses of propranolol. Clin Pharmacol Drug Dev. 2020;9(5):629-638. doi:10.1002/cpdd.768

28. Pearlman EM, Wilbraham D, Dennehy EB, et al. Effects of lasmiditan on simulated driving performance: results of two randomized, blinded, crossover studies with placebo and active controls. Hum Psychopharmacol. 2020;35(5):e2732. doi:10.1002/hup.2732

29. Tsai M, Nery ESM, Kerr L, et al. Pharmacokinetics, safety, and tolerability of lasmiditan in pediatric patients with migraine. Clin Pharmacokinet. 2021;60(6):819-828. doi:10.1007/s40262-020-009 66-z

30. Scott LJ. Ubrogepant: first approval. Drugs. 2020;80(3):323-328. doi:10.1007/s40265-020-01264-5

31. Chiang CC, VanderPluym JH. Ubrogepant in the acute management of migraine: a narrative review. J Pain Res. 2021;14:1185-1192. doi:10.2147/JPR.S244249

32. Voss T, Lipton RB, Dodick DW, et al. A phase IIb randomized, double-blind, placebo-controlled trial of ubrogepant for the acute treatment of migraine. Cephalalgia. 2016;36(9):887-898. doi:10.11 $77 / 0333102416653233$

33. Lipton RB, Dodick DW, Ailani J, et al. Effect of ubrogepant vs placebo on pain and the most bothersome associated symptom in the acute treatment of migraine: the ACHIEVE II randomized clinical trial. JAMA. 2019;322(19):1887-1898. doi:10.1001/jama.2019.16711 
34. Dodick DW, Lipton RB, Ailani J, et al. Ubrogepant for the treatment of migraine. $N$ Engl J Med. 2019;381(23):2230-2241. doi:10.1056/ NEJMoa1813049

35. Yang Y, Chen M, Sun Y, Gao B, Chen Z, Wang Z. Safety and efficacy of ubrogepant for the acute treatment of episodic migraine: a meta-analysis of randomized clinical trials. CNS Drugs. 2020;34 (5):463-471. doi:10.1007/s40263-020-00715-7

36. Ailani J, Lipton RB, Hutchinson S, et al. Long-term safety evaluation of ubrogepant for the acute treatment of migraine: phase 3, randomized, 52-week extension trial. Headache. 2020;60(1):141-152. doi:10.1111/head. 13682

37. Hutchinson S, Silberstein SD, Blumenfeld AM, et al. Safety and efficacy of ubrogepant in participants with major cardiovascular risk factors in two single-attack phase 3 randomized trials: ACHIEVE I and II. Cephalalgia. 2021;3331024211000311. doi:10.1177/03331024211000311

38. Goadsby PJ, Tepper SJ, Watkins PB, et al. Safety and tolerability of ubrogepant following intermittent, high-frequency dosing: randomized, placebo-controlled trial in healthy adults. Cephalalgia. 2019;39(14):1753-1761. doi:10.1177/0333102419869918

39. Jakate A, Blumenfeld AM, Boinpally R, et al. Pharmacokinetics and safety of ubrogepant when coadministered with calcitonin gene-related peptide-targeted monoclonal antibody migraine preventives in participants with migraine: a randomized Phase $1 \mathrm{~b}$ drug-drug interaction study. Headache. 2021;61(4):642-652. doi:10.1111/head.14095

40. Jakate A, Boinpally R, Butler M, et al. Evaluation of the pharmacokinetic interaction and safety of ubrogepant coadministered with acetaminophen or nonsteroidal anti-inflammatory drugs: a randomized trial. Cephalalgia Rep. 2020;3:251581632092118. doi:10.1177/2515816320921186

41. Jakate A, Boinpally R, Butler M, Lu K, McGeeney D, Periclou A. Evaluation of the pharmacokinetic interaction of ubrogepant coadministered with sumatriptan and of the safety of ubrogepant with triptans. Headache. 2020;60(7):1340-1350. doi:10.1111/head.13862

42. Li -C-C, Palcza J, Xu J, et al. The effect of multiple doses of ubrogepant on the pharmacokinetics of an oral contraceptive in healthy women: results of an open-label, single-center, two-period, fixed-sequence study. Cephalalgia Rep. 2020;3:251581632090508. doi:10.1177/2515816320905082

43. Scott LJ. Rimegepant: first approval. Drugs. 2020;80(7):741-746. doi:10.1007/s40265-020-01301-3

44. DeFalco AP, Lazim R, Cope NE. Rimegepant orally disintegrating tablet for acute migraine treatment: a review. Ann Pharmacother. 2021;55(5):650-657. doi:10.1177/1060028020954800

45. Marcus R, Goadsby PJ, Dodick D, Stock D, Manos G, Fischer TZ. BMS-927711 for the acute treatment of migraine: a double-blind, randomized, placebo controlled, dose-ranging trial. Cephalalgia. 2014;34(2):114-125. doi:10.1177/0333102413500727

46. Croop R, Goadsby PJ, Stock DA, et al. Efficacy, safety, and tolerability of rimegepant orally disintegrating tablet for the acute treatment of migraine: a randomised, phase 3, double-blind, placebo-controlled trial. Lancet. 2019;394(10200):737-745. doi:10.1016/S0140-6736(19)31606-X

47. Lipton RB, Croop R, Stock EG, et al. Rimegepant, an oral calcitonin gene-related peptide receptor antagonist, for migraine. $N$ Engl J Med 2019;381(2):142-149. doi:10.1056/NEJMoa1811090

48. Gao B, Yang Y, Wang Z, et al. Efficacy and safety of rimegepant for the acute treatment of migraine: evidence from randomized controlled trials. Front Pharmacol. 2020;10:1577. doi:10.3389/ fphar.2019.01577

49. Negro A, Martelletti P. Gepants for the treatment of migraine. Expert Opin Investig Drugs. 2019;28(6):555-567. doi:10.1080/13543784. 2019.1618830
50. Croop R, Lipton RB, Kudrow D, et al. Oral rimegepant for preventive treatment of migraine: a phase $2 / 3$, randomised, double-blind, placebo-controlled trial. Lancet. 2021;397(10268):51-60. doi:10. 1016/S0140-6736(20)32544-7

51. de Vries T, Al-Hassany L, MaassenVanDenBrink A. Evaluating rimegepant for the treatment of migraine. Expert Opin Pharmacother. 2021;22(8):973-979. doi:10.1080/14656566.2021.1895749

52. Croop R, Ivans A, Anderson MS, et al. A phase 1 randomized study of hemodynamic effects and pharmacokinetic interactions during concomitant use of rimegepant and sumatriptan in healthy adults. Cephalalgia Rep. 2021;4:251581632110079. doi:10.1177/25158163211007922

53. Berman G, Croop R, Kudrow D, et al. Safety of rimegepant, an oral CGRP receptor antagonist, plus CGRP monoclonal antibodies for migraine. Headache. 2020;60(8):1734-1742. doi:10.1111/ head. 13930

54. van Hoogstraten WS, MaassenVanDenBrink A. The need for new acutely acting antimigraine drugs: moving safely outside acute medication overuse. J Headache Pain. 2019;20(1):54. doi:10.1186/ s10194-019-1007-y

55. De Felice M, Ossipov MH, Wang R, et al. Triptan-induced latent sensitization: a possible basis for medication overuse headache. Ann Neurol. 2010;67(3):325-337. doi:10.1002/ana.21897

56. Belanger S, Ma W, Chabot JG, Quirion R. Expression of calcitonin gene-related peptide, substance $\mathrm{P}$ and protein kinase $\mathrm{C}$ in cultured dorsal root ganglion neurons following chronic exposure to $\mathrm{mu}$, delta and kappa opiates. Neuroscience. 2002;115(2):441-453. doi:10.1016/ S0306-4522(02)00452-9

57. Yisarakun W, Chantong C, Supornsilpchai W, et al. Up-regulation of calcitonin gene-related peptide in trigeminal ganglion following chronic exposure to paracetamol in a CSD migraine animal model. Neuropeptides. 2015;51:9-16. doi:10.1016/j.npep.2015.03.008

58. Yan H, Yu LC. Expression of calcitonin gene-related peptide receptor subunits in cultured neurons following morphine treatment. Neurosci Lett. 2013;544:52-55. doi:10.1016/j.neulet.2013.03.040

59. Kopruszinski CM, Xie JY, Eyde NM, et al. Prevention of stress- or nitric oxide donor-induced medication overuse headache by a calcitonin gene-related peptide antibody in rodents. Cephalalgia. 2017;37(6):560-570. doi:10.1177/0333102416650702

60. Tepper S, Ashina M, Reuter U, et al. Safety and efficacy of erenumab for preventive treatment of chronic migraine: a randomised, double-blind, placebo-controlled phase 2 trial. Lancet Neurol. 2017;16(6):425-434. doi:10.1016/S1474-4422(17)30083-2

61. Silberstein SD, Cohen JM, Seminerio MJ, Yang R, Ashina S, Katsarava Z. The impact of fremanezumab on medication overuse in patients with chronic migraine: subgroup analysis of the HALO CM study. J Headache Pain. 2020;21(1):114. doi:10.1186/s10194020-01173-8

62. Dodick DW, Doty EG, Aurora SK, et al. Medication overuse in a subgroup analysis of phase 3 placebo-controlled studies of galcanezumab in the prevention of episodic and chronic migraine. Cephalalgia. 2021;41(3):340-352. doi:10.1177/0333102420966658

63. Scheffler A, Messel O, Wurthmann S, et al. Erenumab in highly therapy-refractory migraine patients: first German real-world evidence. J Headache Pain. 2020;21(1):84. doi:10.1186/s10194020-01151-0

64. Lambru G, Hill B, Murphy M, Tylova I, Andreou AP. A prospective real-world analysis of erenumab in refractory chronic migraine. J Headache Pain. 2020;21(1):61. doi:10.1186/s10194-020-01127-0

65. Cainazzo MM, Baraldi C, Ferrari A, Lo Castro F, Pani L, Guerzoni S. Erenumab for the preventive treatment of chronic migraine complicated with medication overuse headache: an observational, retrospective, 12-month real-life study. Neurol Sci. 2021;42(10):4193-4202. doi:10.1007/s10072-021-05105-5 
66. Saengjaroentham C, Strother LC, Dripps I, et al. Differential medication overuse risk of novel anti-migraine therapeutics. Brain. 2020;143 (9):2681-2688. doi:10.1093/brain/awaa211

67. Navratilova E, Behravesh S, Oyarzo J, Dodick DW, Banerjee P, Porreca F. Ubrogepant does not induce latent sensitization in a preclinical model of medication overuse headache. Cephalalgia. 2020;40(9):892-902. doi:10.1177/0333102420938652

68. Rau JC, Navratilova E, Oyarzo J, et al. Evaluation of LY573144 (lasmiditan) in a preclinical model of medication overuse headache. Cephalalgia. 2020;40(9):903-912. doi:10.1177/0333102420920006
69. Lee MJ, Lee SY, Cho S, Kang ES, Chung CS. Feasibility of serum CGRP measurement as a biomarker of chronic migraine: a critical reappraisal. J Headache Pain. 2018;19(1):53. doi:10.1186/s10194018-0883-x

70. Munksgaard SB, Ertsey C, Frandsen E, Bendtsen L, Tekes K, Jensen RH. Circulating nociceptin and CGRP in medication-overuse headache. Acta Neurol Scand. 2019;139(3):269-275. doi:10.1111/ ane. 13053

\section{Publish your work in this journal}

Drug, Healthcare and Patient Safety is an international, peer-reviewed open-access journal exploring patient safety issues in the healthcare continuum from diagnostic and screening interventions through to treatment, drug therapy and surgery. The journal is characterized by the rapid reporting of reviews, original research, clinical, epidemiological and post-marketing surveillance studies, risk management, health literacy and educational programs across all areas of healthcare delivery. The manuscript management system is completely online and includes a very quick and fair peer-review system. Visit http://www.dovepress.com/testimonials.php to read real quotes from published authors. 